\title{
Las aulas multigrado en el medio rural en Andalucía. Visiones docentes
}

\author{
Multigrade classrooms in rural areas in Andalusia. Teaching perspectives \\ Blas González Alba \\ Pablo Cortés González \\ Analia Leite Méndez
}

\begin{abstract}
RESUMEN
El aula multigrado es una de las señas de identidad de las escuelas rurales, sus características y singularidades pedagógicas y organizativas la convierten en un espacio educativo y escolar particular y heterogéneo. A través de entrevistas abiertas y grupos de discusión recuperamos las voces del profesorado de educación infantil y primaria que desarrolla su práctica en centros educativos ubicados en entornos rurales. El objetivo principal de la investigación es conocer las potencialidades y debilidades de las aulas multigrados a partir de diversas experiencias docentes. Los resultados revelan las potencialidades contextuales, pedagógicas y didácticas inherentes al aula multigrado, mostrando una escuela rural inclusiva, cooperativa y abierta a las nuevas tecnologías y a las metodologías didácticas, pero también nos acercan a conocer las debilidades de la escuela rural, relacionadas principalmente con la dotación de recursos humanos y materiales y la formación inicial y continua del profesorado, revelándose como una escuela poco conocida por los docentes noveles, por los estudiantes de magisterio y por el profesorado universitario.
\end{abstract}

Palabras claves: entrevistas semiestructuradas, escuela rural, práctica docente.

\section{ABSTRACT}

The multigrade classroom is one of the hallmarks of rural schools, its pedagogical and organizational characteristics, as well as singularities, make it a particular and heterogeneous educational space. Through open interviews and discussion groups, we recover the voices of pre-school and primary education teachers who develop their practice in educational centers located in rural settings. The main objective of the research is to know the capabilities and weaknesses of multigrade classrooms based on various teaching experiences. The results reveal the contextual, pedagogical, and didactic capabilities inherent to the multigrade classroom, showing an inclusive rural school, cooperative and open to new technologies and didactic methodologies, but they also bring us closer to knowing the weaknesses of the rural school, related mostly to the endowment of human and material resources and the initial and continuous training of the teaching staff, revealing itself as a little-known school by novice teachers, teaching students and university teaching staff.

Keywords: semi-structured interviews, rural school, teaching practice. 


\section{INTRODUCCIÓN}

Los espacios rurales se caracterizan en gran medida por ser zonas con una baja densidad demográfica, no obstante, desde hace algunos años ha comenzado a emerger un nuevo fenómeno de vuelta a lo rural por parte de personas retornadas, inmigrantes y/o neo-rurales -individuos atraídos por las posibilidades empresariales de la zona(Bustos, 2009), que están reconfigurando el medio rural y reactivando el desarrollo social, demográfico y económico de algunas de estas áreas.

Este aumento de la población residente en contextos rurales ha influido de un modo positivo y notable en el mantenimiento y configuración actual de las escuelas rurales (Abós, 2014), pues su conservación y mejora por parte de las administraciones educativas responde a su capacidad de ofrecer una respuesta escolar eficaz en zonas geográficas dispersas, alejadas de centros urbanos y con poca población, evitar el aislamiento sociocultural de estas poblaciones y disminuir el descenso demográfico de estas zonas rurales (Champollion, 2011).

Aunque denominadas bajo diferentes términos -Colegios Públicos Rurales, Centros Rurales Agrupados, Zonas Escolares Rurales o Escuelas Rurales (Boix y Bustos, 2014)-, las escuelas españolas ubicadas en entornos rurales están sujetas a la misma normativa que las escuelas urbanas, sin embargo, estas cuentan con una serie de peculiaridades -sociales, políticas, económicas y educativas-inherentes a los contextos en las que se encuentran inmersas (Abós, 2007; Ortega, 1995). Aunque las características particulares de estos contextos van a delimitar el propio concepto de "escuela rural", de un modo generalizado esta se puede definir como aquella que está situada en núcleos poblacionales dispersos, con baja concentración de escolares (Martínez y Bustos, 2011), única en la localidad, que presenta la modalidad multigrado

\footnotetext{
Blas González Alba. Miembro del grupo de investigación HUM-619 de la Junta de Andalucía, España. Es doctor en Ciencias de la Educación por la Universidad de Málaga. Sus líneas de investigación están vinculadas con la atención a la diversidad, la resiliencia y la educación desde un enfoque narrativo. Actualmente compagina la docencia como maestro de Pedagogía Terapéutica con la investigación educativa como dos actividades que permiten mejorar su práctica educativa. Correo electrónico: blas@uma. es. ID: https://orcid.org/0000-0002-4769-6522.
}

Pablo Cortés González. Profesor Titular del Departamento de Didáctica y Organización Escolar de la Universidad de Málaga, España. Es miembro del Grupo de Investigación ProCIE-HUM619- de la Junta de Andalucía. Sus líneas de investigación versan en torno a las narrativas, la identidad profesional de los agentes educativos, la resiliencia, las prácticas disruptivas y las ecologías del aprendizaje, teniendo un centenar de artículos y capítulos de libros sobre dichas temáticas. Ha sido coordinador del proyecto de innovación docente titulado "El uso de narrativas en la enseñanza universitaria. Experiencias y relatos (auto) biográficos para la construcción del conocimiento social, científico y profesional”. Correo electrónico: pcortes@uma.es. ID: https://orcid.org/0000-0002-9604-044X.

Analia Leite Méndez. Profesora del Departamento de Didáctica y Organización Escolar de la Universidad de Málaga, España. Es doctora en Educación e investigadora del Grupo Procie (HUM619). Ha publicado artículos y textos en el ámbito de la formación del profesorado, identidades docentes, voluntariado y formación docente desde una perspectiva narrativa-biográfica. Correo electrónico: aleite@uma.es. ID: https://orcid.org/0000-0001-5064-999X. 
y que está situada en poblaciones que no superan los 500 habitantes (Corchón, 2001, 2005). En este escenario es, quizás, el aula multigrado y todo su complejo entramado pedagógico (metodológico, organizativo...) una de las señas de identidad de las escuelas rurales (Boix, 2011).

\section{La escuela rural en el contexto nacional e internacional}

A pesar de que tradicionalmente la escuela rural ha sido denostada social, política y económicamente en comparación con la llamada "escuela urbana", resulta clarificador señalar dos dimensiones que son claves para entender la importancia que tiene la escuela rural en el panorama internacional y en ciertas regiones españolas.

Por un lado, es significativo considerar que la escuela primaria rural multigrado representó durante el curso 2014/2015 en países como México o El Salvador el 43.8 y el 55\%, respectivamente, de las escuelas primarias totales (Juárez, 2017; Juárez, Vargas y Vera, 2015). En el caso de Argentina la escuela rural constituye el 37\% (nivel inicial), el 47\% (nivel primario) y el 26\% (nivel secundario) de sus instituciones escolares (Boletín Diniece, 2015). Así mismo, y considerando la situación de otros países latinoamericanos, la escuela rural básica o primaria representa respecto al total de sus instituciones escolares el 36.6\% en Chile y el 76.1\% en Perú (2012), según los datos aportados por el informe temático 2015/2016 elaborado por el gobierno peruano. Con unos datos similares, el Informe de escuela rural y programa de política y confianza comunitaria elaborado por Showalter, Klein, Johnson y Hartman (2017), señala que la escuela rural en Estados Unidos constituyó el 28.5\% del total de los centros escolares y atendió a más de siete millones de estudiantes durante el curso escolar 2015/16.

En el caso de España y según la información aportada por el Ministerio de Educación y Formación Profesional del Gobierno (informe 2019 sobre el estado del sistema educativo), para el curso 2017/2018 se escolarizaron 71.727 alumnos y alumnas en los centros rurales de educación infantil (3-5 años) y educación primaria (6-11 años), representando el 0.9\% del total de alumnado matriculado. No obstante, y como se muestra en la tabla 1, en algunas comunidades autónomas, como ocurre en Andalucía, donde se ubica esta investigación, esta proporción es bastante más alta debido tanto a las dimensiones espaciales como a las características geográficas que presentan algunas de estas comunidades autónomas.

Por otro lado, y considerando lo que diversas investigaciones muestran, la valoración de las debilidades y fortalezas que presentan las escuelas rurales multigrado nos ofrece una panorámica más delimitada y concreta de sus necesidades y virtudes. En este sentido, y centrándonos en el caso de España, recurrimos al trabajo realizado por Feu (2004), cuyos resultados muestran la escasez de recursos materiales, infraestructuras y profesorado como las principales demandas de los docentes que 
Tabla 1. Información de los centros rurales españoles agrupados por comunidades con mayor representación.

\begin{tabular}{cccc}
\hline Comunidad autónoma & Centros & Unidades & Alumnado y porcentaje total de alumnado matriculado en escuelas rurales \\
\hline Andalucía & 107 & 1.117 & $10.993(15,32 \%)$ \\
\hline Aragón & 75 & 783 & $8.496(11,84 \%)$ \\
\hline Castilla y León & 181 & 1.463 & $15.156(21,13)$ \\
\hline Castilla-La Mancha & 77 & 838 & $8.096(11,28)$ \\
\hline Cataluña & 86 & 806 & $10.481(14,61 \%)$ \\
\hline
\end{tabular}

Fuente: Informe 2019 sobre el estado del sistema educativo (Ministerio de Educación y Formación Profesional del Gobierno de España).

trabajan en contextos rurales. Sin embargo, estos aluden también a su capacidad para desarrollar pedagogías activas, generar espacios y tiempos de atención personalizada al alumnado, flexibilizar el tiempo y el calendario, atender de un modo efectivo y proactivo a la diversidad y utilizar sistemas de evaluación integrales, como aspectos positivos a considerar (Feu, 2004). En este sentido, otros autores apuntan al respecto que la escuela rural multigrado en España se configura como un espacio de innovación pedagógica (Abós, 2014), que permite vincular los contenidos con el contexto, establecer relaciones docente-alumnado más estables y positivas (Bustos, 2007) y crear un microsistema social de aula que potencia la capacidad de autonomía de aprendizaje en el alumnado (Boix, 2011).

Así mismo, Champollion (2011) señala algo similar respecto a las escuelas rurales multigrado francesas, para ello destaca su capacidad de individualizar los procesos de enseñanza-aprendizaje, de fomentar la autonomía del alumnado y potenciar la monitorización del alumnado por parte de otros compañeros y compañeras.

Si centramos la mirada en Latinoamérica encontramos una situación parecida, en este sentido, las escuelas rurales multigrado mexicanas presentan limitaciones relacionadas con las infraestructuras, la supervisión, la gestión escolar, la formación docente, la escasez de supervisores, asesores técnicos pedagógicos y docentes especialistas (Juárez, 2017), a lo que se le suma una excesiva carga burocrática que limita el tiempo para atender a todo el alumnado (Juárez, Vargas y Vera, 2015). Esta situación coincide en gran medida con el panorama que encontramos en las escuelas rurales multigrado de El Salvador, al respecto Juárez (2017) señala que la escasa disponibilidad de material didáctico de apoyo, de libros de textos, infraestructuras, mobiliario, personal técnico y de apoyo y las limitaciones en el acceso a recursos tecnológicos e internet son factores que van a limitar en gran medida los procesos de aprendizaje, sin embargo, el citado autor también hace referencia a una escuela rural que ofrece a los docentes mayor grado de libertad, autonomía pedagógica, crecimiento y desarrollo profesional, y una mayor aproximación y mejor conocimiento del alumnado, de las familias y del contexto. 


\section{La organización de las aulas multigrado}

La estructuración espacial y organizativa del aula es uno de los factores claves en los procesos de enseñanza-aprendizaje puesto que el aula es "desde un punto de vista formal de la educación, el lugar donde se desarrollan principalmente los procesos de enseñanza-aprendizaje" (Cortés, González y Sánchez, 2018, p. 76) y, en este sentido, aspectos como su organización y recursos van a configurar los procesos educativos y las relaciones sociales y educativas que se desarrollan en la misma (Leite, 2011).

La organización escolar en aulas multigrados, multinivel, internivel, mixto, niveles compartidos, multiedad o multicurso (Bustos, 2010, 2013) se ha convertido en una peculiaridad, en un símbolo y en una respuesta organizativa y curricular a esa ruralidad (Boix, 2011; Boix y Bustos, 2014; Bustos, 2010; Corchón, 2000). No obstante, y aunque la multigraduación sea "la característica más singular de la escuela rural" (Boix y Bustos, 2014, p. 32), este modo de organización del aula no es exclusivo de las denominadas "escuelas rurales", pues encontramos también otros centros educativos ubicados en contextos urbanos que se organizan en aulas multigrado (Ruiz y Ruiz, 2017), como un modo de organización, funcionamiento y mantenimiento de centros escolares (Sepúlveda y Gallardo, 2011) que presentan unos niveles bajos de matriculación (Bustos, 2010).

Como señala Jiménez (1983), estas aulas se caracterizan principalmente porque "en una misma clase conviven niños y niñas de distintas edades y niveles de escolaridad" (p. 13), es decir, en ellas se agrupa y combina alumnado de distintos niveles (Quílez y Vázquez, 2012) y/o grados educativos (Bustos, 2010, 2013; Cantón, 2004; Hinojo, Raso e Hinojo, 2010), se atiende a alumnado con diferente grado de conocimiento, cuenta con una ratio alumno/a-maestro/a baja (Cantón, 2004; Sauras, 2000) y se acerca al modo más natural de encuentro y trabajo entre escolares (Abós y Boix, 2017).

Como apuntan Quílez y Vázquez (2012), desde una perspectiva organizativa y considerando el número de estudiantes encontramos principalmente dos tipos de aulas multigrado, las "aulas unitarias", en las que se encuentra alumnado de diferentes grados educativos con un único docente en el aula (Boix y Bustos, 2014; Ortega, 1995), y por tanto en la escuela, y las “aulas graduadas incompletas", en las cuales hay varios niveles educativos donde encontramos a un docente por aula.

\section{Metodología}

El presente trabajo se enmarca en la línea temática de las investigaciones de corte cualitativo realizadas por Boix y Bustos (2014) -entrevistas semiestructuradas- y Bustos (2007) -estudios de caso-, no obstante, en esta ocasión hemos recurrido a un estudio de casos colectivo (Stake, 1998) con el objetivo de investigar e indagar la experiencia docente a partir de la revalorización de las voces y las experiencias profesionales del profesorado participante. 
Como venimos señalando, son muchos los estudios (Abós, 2014; Boix, 2011; Bustos, 2007; Champollion, 2011; Feu, 2004; Juárez, Vargas y Vera, 2015; Juárez, 2017; Sepúlveda y Gallardo, 2011) que nos muestran las potencialidades (mayor autonomía docente y pedagógica, ratio alumnado-profesorado baja, individualización de la enseñanza...) y debilidades (escasez de recursos humanos y materiales, limitación de especialistas, inestabilidad de la plantilla...) de las escuelas multigrados ubicadas en los medios rurales. No obstante, y con el objetivo de conocer aspectos menos destacados como su organización-reorganización interna, la asignación de recursos humanos y económicos, las responsabilidades organizativas y burocráticas del profesorado, cómo se organizan los procesos de formación específicos del profesorado, entre otros aspectos, hemos desarrollado este trabajo. En este sentido, la investigación tiene como propósito principal conocer las fortalezas y debilidades de las aulas rurales multigrado. De un modo más específico y considerando los aspectos anteriormente reseñados se han concretado los siguientes objetivos:

1. Indagar la experiencia docente en las aulas multigrado en el entorno rural.

2. Identificar las necesidades y posibilidades educativas de las escuelas rurales que se organizan en este tipo de aulas.

3. Rescatar propuestas de mejora para las mencionadas aulas.

\section{Participantes}

La selección de los participantes responde a un muestreo intencional por conveniencia

y criterial. Para garantizar la idoneidad de los participantes se ha considerado una serie de criterios para su selección: 1) docentes que trabajen en la actualidad en una escuela rural, 2) diversidad de años de experiencia y género, 3) que estén representadas todas las especialidades.

En el estudio han participado 16 docentes que imparten clases en seis centros escolares públicos situados en contextos rurales de la provincia de Málaga (cuatro) y Granada (dos), de los cuales cuatro de ellos son denominados CEIP (Centro de Enseñanza Infantil y Primaria) y los otros dos CPR (Colegio Público Rural). Aunque ambos tipos de centros se organizan en aulas multigrados, encontramos cinco centros que se organizan como aulas graduadas incompletas y uno que se organiza como “aula unitaria” (Quílez y Vázquez, 2012).

Por especialidades han participado en la investigación tres maestras de educación infantil (una de ellas directora), cuatro maestros de educación infantil y primaria (en aula unitaria), nueve maestros y maestras de educación primaria y especialidades, entre los cuales encontramos a dos maestros de educación física (uno director), una maestra de música y primaria (directora), cinco maestros y maestras de lengua extranjera (cuatro de inglés y uno de francés) y una maestra de pedagogía terapéutica (itinerante). 
Tabla 2. Información de los centros, especialidad y experiencia como docentes en la escuela rural del profesorado participante.

\begin{tabular}{cccc}
\hline Nombre & Centro & Especialidad & Experiencia en escuela rural \\
\hline C.G.R. & CEIP M.H. & Infantil (Directora) & 9 años y 5 meses \\
\hline E. C. & CEIP M.H. & Inglés y Primaria & 6 meses (interino ${ }^{1}$ \\
\hline A.G.M. & Compartida entre CEIP M.H y R.A. & Pedagogía Terapéutica & 1 año y 5 meses \\
\hline S. V. C. & CEIP M.H. & Infantil y Primaria & 4 años y 7 meses \\
\hline F. A. & CEIP M.H. & Educación Física, Primaria (Director) & 1 año y 5 meses \\
\hline B.L.P. & CEIP R.A. & Inglés y Primaria (Directora) & 7 años y 6 meses \\
\hline M. A. & CEIP R.A. & Francés y Primaria & 5 meses (interina) \\
\hline J.R. & CPR L.A. & Infantil y Primaria (Director) & 7 meses \\
\hline I.D. H. & CPR E. B. & Infantil (Director) & 1 años y 7 meses \\
\hline P. C. G. & CEIP M. Z. & Primaria e Inglés & 7 meses (Interino) \\
\hline M.E.E. & CEIP M. Z. & Infantil & 2 años y 6 meses \\
\hline F. G. A. & CEIP M. Z. & Música y Primaria (directora) & 2 años y 4 meses \\
\hline N. G. & CEIP L. F. & Infantil & 5 meses (Interina) \\
\hline R.E. & CEIP L. F. & Infantil y Primaria & 2 años y 5 meses \\
\hline E.A. & CEIP L. F. & Educación Física y Primaria & 2 años y 6 meses \\
\hline R.R. & CEIP L. F. & Primaria e inglés (Director) & 6 años y 5 meses \\
\hline
\end{tabular}

Profesorado que no tiene un destino definitivo y que por tanto sustituye temporalmente a un docente.

Fuente: Elaboración propia.

\section{Estrategias, procedimiento e instrumentos}

Con el objeto de acercarnos a la realidad social, escolar y educativa a la que se enfrentan los maestros y maestras que imparten docencia en aulas multigrado, les hemos planteado dos cuestiones de partida:

(a) Relata cómo es tu experiencia en el aula multigrado.

(b)Debilidades y fortalezas del aula multigrado a partir de tu experiencia docente.

A lo largo del curso escolar 2018/19 se han realizado dieciséis entrevistas individuales y abiertas con una duración promedio de 50 minutos en las que el profesorado ha relatado sus vivencias, percepciones y experiencias educativas en el aula multigrado. No obstante, a lo largo del transcurso de las entrevistas han emergido preguntas que han permitido aclarar y/o profundizar en algunas de las cuestiones planteadas en los relatos.

Como procedimiento metodológico, cabe mencionar que una vez transcritas las entrevistas se ha procedido a la devolución oral e individual de las mismas, este hecho ha generado un segundo encuentro con una duración aproximada de 70 minutos que ha permitido -en algunos casos- realizar cambios y aclaraciones sobre fragmentos confusos y/o que emerjan otras experiencias que han complementado la información obtenida durante la primera entrevista. 
Finalmente (junio y julio de 2019) se ha realizado un grupo de reflexión y discusión con siete de los y las docentes participantes, en dos sesiones de 90 minutos. En este encuentro se han puesto en común principalmente cuestiones relacionadas con las potencialidades, debilidades y propuestas de mejora de las escuelas ubicadas en entornos rurales.

Con el objeto de considerar una serie de cuestiones éticas relacionadas tanto con el ejercicio profesional docente como con el proceso investigador, todo el procedimiento investigador se ha desarrollado durante periodo no lectivo, se han empleado abreviaturas con la intención de preservar el anonimato del profesorado, se ha contado con su consentimiento informado y tras la finalización y transcripción de las entrevistas se les ha devuelto las mismas al profesorado.

\section{Análisis de las entrevistas}

$\mathrm{El}$ análisis de los relatos ha transitado desde una lógica singular -considerando las categorías individuales, intracaso- hacia una lógica transversal -intercaso- en la que han emergido categorías comunes y coincidentes (Cornejo, Rojas y Mendoza, 2008).

Toda la información obtenida durante las entrevistas, las devoluciones y los grupos de discusión se ha analizado a mano y simultáneamente por parte de los tres autores del artículo, posteriormente se han mantenido dos encuentros en los que se han discutido las categorías emergentes. Del análisis de los relatos biográficos ha emergido una serie de cuestiones comunes a muchos de los relatos, de las que destacamos para este artículo:

- Realidad docente y escuela rural: debilidades y fortalezas.

- Dotación de recursos materiales y mejora de las infraestructuras, un asunto pendiente.

- La formación inicial y continua: una tarea compartida y olvidada.

- La complejidad de las lógicas en las aulas multinivel.

A raíz de los relatos de los y las docentes se han identificado, como hemos apuntado anteriormente, cuatro categorías emergentes que giran en torno a la experiencia docente en las aulas multigrados. A continuación procedemos a profundizar en las mencionadas categorías de análisis los resultados obtenidos en forma de evidencias (fragmentos de las entrevistas, devoluciones y grupos de discusión).

\section{Realidad docente y escuela rural: debilidades y fortalezas}

$\mathrm{El}$ profesorado constituye un recurso fundamental con el que cuentan las escuelas; su asignación, que es responsabilidad de las administraciones educativas, está determinada principal y exclusivamente por criterios cuantitativos -número de alumnado del centro-, aspecto que perjudica a las escuelas ubicadas en entornos rurales, puesto que una de sus particularidades es el aula multigrado y una ratio alumnado-docente 
baja (Cantón, 2004). Este hecho, que es advertido por la mayor parte de los y las docentes, afecta de un modo generalizado a la organización escolar de cada centro y aula, pues ante la ausencia de cualquier maestro o maestra - por enfermedad, reuniones de coordinación, asistencia a formación en planes y proyectos, reuniones...- se tienen que reestructurar y reorganizar las aulas debido a la falta de profesorado. Esta situación, preocupante y que repercute de un modo significativo en los procesos de aprendizaje del alumnado, no ocurre en los centros urbanos, pues estos, al contar con mayor número de alumnado y por tanto con mayor asignación docente, disponen de profesorado de refuerzo educativo - uno, dos o tres docentes por centro en función del número de alumnado- que ejerce estas funciones de sustitución.

Cuando falta alguien es un incordio, sobre todo cuando falta la maestra de infantil y tienes que meter a los pequeños con los de primaria. Los de tres años son distintos y la diferencia de edad se nota a la hora de impartir clase y tenerlos todos juntos [Grupo de discusión, E.C., maestro de primaria e inglés, interino y primer año en escuela rural multigrado].

Veo negativo el tener que ir mendigando a recursos humanos el tema de que necesitamos otro profesor cuando deberían ser ellos los que nos dan el recurso. Contando con que un centro tan pequeño, uno se pone malo y la otra persona se tiene que quedar con veinte alumnos desde tres años hasta sexto de primaria todos juntos. Si contáramos con tres personas en la plantilla, aunque la ratio sea baja, pues ese problema no existiría [Grupo de discusión, C.G.R., directora y maestra de infantil, más de nueve años en escuela rural multigrado].

Como señalan ambos docentes, la escasez de profesorado en los centros rurales multigrados provoca a menudo situaciones en las que encontramos a un solo docente que tiene que atender de forma continua a todo el alumnado que cursa educación infantil y primaria, aspecto que potencia la sensación de aislamiento y soledad tan asociada a la escuela ubicada en contextos rurales (Quílez y Vázquez, 2012). Este hecho es interpretado (1) como una situación injusta y un "incordio", por parte de un docente sin experiencia en una escuela rural multigrado, pues es la primera vez que se enfrenta a este tipo de situaciones, y (2) como una reivindicación histórica a la administración educativa, por parte de una maestra que asume la dirección del centro y su organización escolar desde hace más de nueve años.

Esta situación de soledad y aislamiento a la que aludíamos anteriormente es aún más dramática cuando ocurre en una escuela rural con un solo docente, como describe uno de los maestros: "Uno de los grandes problemas es que como soy el único maestro, cuando este año por motivos de salud no he podido ir el colegio este no se ha abierto" (entrevista J.R., único maestro del centro), es decir, asistimos al cierre temporal de una escuela, situación que genera en las familias una sensación de abandono por parte de las administraciones educativas y de indefensión para el docente, repercutiendo, como veremos más adelante, en su decisión de permanecer un curso más en el centro. 
Así mismo, contar con una plantilla reducida no exime de ciertas responsabilidades administrativas, formativas y pedagógicas vinculadas con la coordinación de diferentes planes y proyectos del centro. Esta situación, a su vez, implica una tendencia hacia la multifuncionalidad del docente del aula rural multigrado que se manifiesta en la asunción de tareas organizativas y responsabilidades de carácter transversal que tiene que desempeñar (reuniones con otros cargos directivos, con inspectores de educación, reuniones de formación y coordinación como responsables de planes y programas educativos...) y que lo obligan a abandonar el centro educativo sin ser sustituido por parte de las administraciones educativas. Estas actividades, que tienen una carga burocrática y formativa obligatoria en aspectos como coeducación, seguridad, educación ambiental..., es decir, no es una formación específica y relacionada con habilidades docentes en aulas multigrados, requieren de una continua reorganización del alumnado en función de estas necesidades. Como se advierte a continuación, este hecho es señalado con incredulidad por un docente recién incorporado a un aula rural multigrado.

Yo soy coordinador de igualdad, coordinador de paz, llevo el plan de autoprotección y esta semana tengo una reunión de paz, la semana que viene y dentro de dos semanas me tengo que ir otra vez. La compañera también es la coordinadora de otras cosas y tenemos que dejar las clases bastantes días porque tenemos que hacer distintas tareas [entrevista, E.C., maestro de primaria e inglés, interino y primer año en escuela rural multigrado].

En relación con la asignación de profesorado especialista en atención a la diversidad ocurre algo similar, una ratio baja repercute de manera significativa en la concesión de sesiones de intervención de los maestros y maestras de pedagogía terapéutica, audición y lenguaje y orientación educativa. Asistimos a prácticas educativas necesarias para atender al alumnado con Necesidades Específicas de Apoyo Educativo (NEAE) que se espacian en el tiempo como consecuencia de compartir los mismos recursos humanos entre diferentes centros educativos.

Dependiendo del número de alumnos, de la ratio, incluyen a un especialista o disminuyen un especialista. Del equipo de orientación pasaba tanto con la maestra de PT [pedagogía terapéutica], como AL [maestro/a de audición y lenguaje], pero la intervención es mínima, un día al mes [entrevista, I.D.H, director, maestro de educación infantil y primaria, único maestro del centro].

Yo estoy compartida entre tres centros, al más grande voy tres días en semana, pero a los otros dos que tienen aulas agrupadas solo voy un día a la semana, y el maestro de AL cada quince días, la verdad que no nos permite tener una continuidad, nuestro trabajo al final se limita a asesorar a los docentes [entrevista, A.G.M., maestra de pedagogía terapéutica].

Que la orientadora venga una vez al mes, muy mal, el AL viene cada quince días y el PT una vez a la semana, eso es para mí muy poco, sobre todo cuando hay necesidades, deberían venir mínimo dos veces o más [Grupo de discusión, B.L.P., directora, maestra de inglés y primaria].

En relación con lo anteriormente señalado, seguimos encontrando centros ubicados en entornos rurales con plantillas incompletas, pues no siempre disponen de 
profesorado especialista. Este hecho repercute en la actividad docente de los maestros y maestras generalistas de educación primaria o de otras especialidades, los cuales tienen que "completar" su horario lectivo impartiendo asignaturas específicas para las que no están formados; como señala una maestra:

Yo no soy especialista en música, no recuerdo casi nada de solfeo y no pueden seguir conmigo la música como deberían, hago lo que puedo. No hay especialista de música y yo cubro esas horas [entrevista, M.A., maestra de francés y primaria].

Estas situaciones, además de generar malestar en los docentes e intrusismo profesional, producen un deterioro en la formación del alumnado, pues generalmente reciben estas materias de un modo teórico, reproductivo y haciendo uso exclusivo del libro de texto puesto que el profesorado no posee aptitudes, habilidades y/o conocimientos para impartir dichas materias.

La distancia que separa a las escuelas rurales de los núcleos urbanos provoca que muchos docentes no escojan estos destinos y estos puestos sean asignados a maestros/as noveles o en régimen de interinidad, dificultando la estabilidad de las plantillas y la formación de un equipo docente estable. Este hecho, que provoca un periodo de incertidumbre en el profesorado y precisa de un proceso de "formación interna y no formal" por parte del resto de docentes con mayor experiencia, ha sido apreciado por dos maestras y directoras de este modo:

Veo como muy negativo la inestabilidad de la plantilla, pues no nos permite una continuidad en la implantación de proyectos. El que año tras año aparezca un nuevo profesorado, venga, llegue, se haga a trabajar con este tipo de agrupamiento y que el alumnado se haga con el profesorado. Además, todos los años tengo que volver a explicar la metodología y cómo se trabaja con este tipo de alumnado, yo lo veo un hándicap importante. También contamos con pocos recursos personales, eso debido a nuestra baja ratio, está claro [entrevista, C.G.R., directora y maestra de infantil].

El problema que tiene este tipo de centros es que todos los años cambian, entonces todos los años vienen personas nuevas, personas con experiencia, personas sin experiencia, y mientras se adaptan al entorno es complicado [grupo de discusión, B.L.P., directora maestra de inglés y primaria].

A pesar de que la mayor parte del profesorado participante tiene experiencia impartiendo docencia en centros rurales, la presencia de un o una docente sin experiencia en el colegio precisa de una mayor carga de trabajo para estos. Como es advertido por el profesorado con mayor experiencia, aspectos como la organización del currículum, del aula o el desarrollo de metodologías específicas han requerido de un proceso formativo específico e informal que ha sido asumido por los compañeros con más experiencia.

Se genera de este modo una situación cíclica de docentes "de paso" que afecta al proceso de enseñanza-aprendizaje y no permite a los centros educativos rurales iniciar o consolidar sus respectivos proyectos educativos (Boix, 2004; Sauras, 2000), 
desarrollar programas o planes específicos (Bustos, 2007) o formar una plantilla estable capaz de realizar una formación específica e implantar proyectos de innovación educativa duraderos, aspectos que en gran medida afectan a los procesos de aprendizaje del alumnado.

\section{Dotación de recursos materiales y mejora de las infraestructuras, un asunto pendiente}

La solicitud de recursos materiales por parte de los docentes de las escuelas ubicadas en el medio rural ha sido una reivindicación histórica (Bustos, 2007). En los últimos años se han producido algunas mejoras en las escuelas rurales -creación y rehabilitación de espacios, dotación de material y recursos tecnológicos, mejora de la red de conexión a internet... (Sauras, 2000)-, sin embargo, seguimos encontrando escuelas rurales cuyas infraestructuras y espacios específicos - biblioteca, zona y patio para el deporte, zona para el recreo-, recursos materiales (Bustos, 2013; Corchón, 2005) y tecnológicos (Bustos, 2007; Hinojo, Raso e Hinojo, 2010) continúan siendo insuficientes, precarios u obsoletos.

No existe un aula específica para trabajar con el alumnado, a mí me gusta trabajar en inclusión, pero en algunas ocasiones necesito realizar actividades específicas y me tengo que ir al pasillo, además si necesito algún tipo de material lo tengo que traer yo. Les pasa lo mismo a los maestros especialistas, el maestro encargado de dar música apenas cuenta con instrumento musicales, en educación física el colegio solo tiene dos porterías de futbol y algunas pelotas [entrevista, A.G.M., maestra de pedagogía terapéutica].

Como señalan Corchón y Raso (2002), las escuelas rurales tienen pocos recursos materiales, este hecho se constata en la dotación de libros para la biblioteca o el aula, instrumentos musicales, material de intervención para trabajar con alumnado diagnosticado con NEAE o material deportivo, entre otros. Este déficit está provocado por: (1) una dotación presupuestaria asignada a cada centro que es proporcional al número de alumnos y alumnas, y (2) que la escasa cantidad de dinero de la que disponen estos centros escolares suele ir para la adquisición de recursos que los responsables (directores y directoras) consideran que son prioritarios o de suprema necesidad para el centro.

El colegio no tiene muchos recursos, yo estoy en la casita donde está el aula de infantil, en otra parte del pueblo hay otra casita para primaria, pero yo estoy sola. El único recurso que tengo es la fotocopiadora [entrevista, M.E.E., maestra de educación infantil].

Carecemos de recursos, por ejemplo, yo no dispongo de pizarra digital, sería muy fácil poner un cañón y un salvapantallas y utilizarlo para todo, es decir, para todas las materias [entrevista, M.A., maestra de francés y primaria].

Hay una gran falta de recursos, no tengo pizarra digital, no tengo internet en el aula, tengo que llevar los programas o videos desde casa [entrevista, R.E., maestro de primaria]. 
En relación con la dotación de recursos materiales, el personal docente hace referencia principalmente a la escasez y/o mal funcionamiento de los recursos TIC. $\mathrm{Al}$ respecto hay que señalar que la entrada en vigor del Decreto 72/2003, de 18 de marzo, de Medidas de Impulso de la Sociedad del Conocimiento en Andalucía ha permitido dotar a las aulas de los centros educativos de Andalucía de recursos TIC, entre las que se encuentran las aulas de las escuelas rurales.

Este proceso paulatino de incorporación de herramientas y recursos vinculados a las TIC (ordenadores, pizarras digitales, tablets, red de banda ancha ultrarrápida...) en las aulas sigue en proceso, no obstante, la demanda de los centros sigue estando por encima de la oferta. En este sentido, son muchos los centros que cuentan con recursos TIC, pero (1) estos -ordenadores, pizarras digitales- están ubicados en aulas específicas y, por lo tanto, solo pueden ser utilizados por el alumnado y por los docentes de esa aula, y (2) los que pueden ser compartidos (tabletas electrónicas y ordenadores portátiles) están deteriorados por su uso, descargados o desactualizados, por lo que caen en el desuso.

No obstante, lo que ponemos en cuestión en estas líneas es si la simple asignación de recursos resuelve las necesidades y problemas educativos de estudiantes y familias en zonas rurales, puesto que el mero uso de tecnología -y cuanto menos obsoletano garantiza una práctica educativa de calidad y relevante para el alumnado. En este sentido pensamos que los recursos deberían entenderse bajo la lógica de lo rural y de los contextos próximos en los que los centros estén inmersos (Cortés, González y Sánchez 2018; Cortés, Simancas y Mañas, 2018).

\section{La formación inicial y continua, una tarea olvidada}

Desde una dimensión formativa, y como manifiesta la investigación realizada por Abós (2011), de los 183 centros de educación superior españoles estudiados que ofertan el grado de educación infantil y primaria y que están vinculados a 39 universidades públicas y privadas, ninguno de ellos hace referencia en la regulación de sus títulos (Orden ECI/3854/2007 y Orden ECI/3857/2007) y de forma explícita a objetivos y/o planificación de enseñanzas relacionadas con la escuela rural. Como señala la autora, y a pesar de que encontramos de un modo implícito objetivos que aluden a la escuela rural con términos como diversidad, grupos heterogéneos, trabajo individual y colectivo de carácter específico, diversidad de acciones de su funcionamiento, múltiples posibilidades de convivencia, entre otros, estos conceptos son muy ambiguos y no dan respuestas formativas a las necesidades reales de los futuros docentes.

En este sentido, y como se desprende de las entrevistas, el profesorado no ha recibido formación inicial o continua específica relacionada con la enseñanza en entornos rurales (organización escolar, metodología...) o ha desarrollado su periodo formativo de prácticas en un colegio rural y/o multigrado. Esta situación, bastante 
significativa, manifiesta una limitación en los planes de estudio y/o un escaso interés por parte de las propias universidades y Centros de Profesorado para ofrecer una formación docente ajustada a las necesidades y potencialidades de la escuela rural multigrado.

La escasa o nula formación para ejercer docencia en estos centros conduce a muchos docentes a enfrentarse a situaciones escolares que desconocen y para las que no han sido formados (Sauras, 2000), como nos describe una maestra:

En la carrera no me hablaron de CPR, a mí no me hablaron de este tipo de centros, es más, yo terminé en junio y en septiembre me mandaron a un CPR, y yo no tenía ni idea [entrevista, S.V.C., maestra de primaria].

En este sentido, el profesorado coincide en señalar la falta de conocimiento del funcionamiento de las escuelas ubicadas en el medio rural, la escasa formación universitaria recibida, la poca oferta formativa y el impacto inicial de impartir clases en un aula multinivel como los aspectos más destacables de su experiencia docente en las aulas rurales multigrados.

A mí no me han formado para este tipo de situaciones, aquí siempre uno va aprendiendo y sigo aprendiendo porque cada día voy aprendiendo cosas [entrevista, E.C., maestro de primaria e inglés, interino y primer año en escuela rural multigrado].

La escasa o nula formación inicial del profesorado en cuestiones organizativas, didácticas y metodológicas relacionadas con la docencia en el aula multigrado los conduce a actuar "según la formación inicial que han recibido desde las universidades o bien repitiendo lo que ellos vivieron en su época de estudiantes de primaria" (Boix, 2011), es decir, a partir del "conocimiento sedimentado" (Rivas, 2014; González, Leite y Rivas, 2018) que han adquirido a lo largo de su experiencia escolar como estudiantes, como expresa una docente:

No hay ningún tipo de formación especial para los maestros que vienen a escuelas rurales o a escuelas con niños agrupados, ni ninguna metodología específica para trabajar con el alumno en sí, cada maestro pues trata de hacerlo lo mejor que sabe o puede. No existe, desde ningún organismo, no existe nada especial dirigido a maestros de escuelas rurales [entrevista, C.G.R., directora y maestra de infantil].

Como señalan diversos autores (Boix, 2004; Bustos, 2007, 2013; Quílez y Vázquez, 2012), la formación inicial del profesorado juega un papel crucial en la práctica docente en el medio rural. A la baja atención que tanto la universidad (Ruiz y Ruiz, 2017) como los centros de profesorado (CEP) dedican a la formación de los y las docentes para trabajar en las escuelas ubicadas en el medio rural se le suma una notable falta de materiales, referencias, textos, investigaciones, producciones científicas y literarias que aporten conocimientos específicos - metodológicos, didácticos, organizativos...- que permitan trabajar en las aulas multigrados (Bustos, 2007, 2011) y conocer esta realidad escolar. 
El desconocimiento de la escuela rural multigrado va a generar un periodo de incertidumbre o "shock ante la realidad" (Veenman, 1984) en los docentes que se enfrentan por primera vez a este tipo de situaciones escolares, de este modo lo expresa un maestro con poca experiencia en la docencia en el ámbito rural:

Este es mi primer año aquí, entonces la formación que uno tiene porque, ¡claro!... es que esto es totalmente distinto a lo que yo estaba acostumbrado a tener, entonces me asusté un poco porque, aunque son pocos niños, lo de las clases juntas me daba un poco de respeto [entrevista, E.C., maestro primaria e inglés, interino y primer año en escuela rural multigrado].

La inexperiencia y desconocimiento acerca de cómo funcionan las escuelas multinivel y de cuáles son sus características y recursos materiales y espaciales conduce a los docentes a comprobar que su conocimiento acerca de la escuela se aleja de la realidad escolar de las escuelas rurales multigrado (Bustos, 2014) y los conduce a experimentar una etapa de transición (Abós, 2007) que será compensada con la experiencia, como señalan algunos docentes:

A mí me llamó la atención el primer día que llegué porque cuando dijeron de salir al recreo, el recreo se hacía en la plaza del pueblo, aquello eran los niños corriendo por la placilla. Me costó en este sentido organizarme el trabajo, pero ya una vez que le coges la rutina... [entrevista, M.E.E., maestra de educación infantil].

Es cierto que los principios nunca son fáciles, y hasta que tú como maestra te adaptas a ellos y ellos a ti pasa un tiempo, pero al final se convierte en una buena dinámica [entrevista, E.A., maestra de educación física y primaria].

Los docentes coinciden en señalar que su experiencia en las aulas multigrados ha transitado por un "eje temporal antes-después" (Bustos, 2007) que se percibe en lo metodológico y organizativo, este comienza con una sensación de "incertidumbre", "reparo" o "respeto" como consecuencia de enfrentarse a una realidad desconocida (Bustos, 2007) que se ha ido disipando con el paso del tiempo y con la incorporación de estrategias educativas basadas en el ensayo-error y en las aportaciones de otros docentes.

Así mismo y considerando este hecho, resulta significativo comprobar cómo doce de los dieciséis docentes que han participado en la investigación continúan seleccionando como destino - provisional o definitivo- escuelas rurales, quizás por muchas de las potencialidades que esta presenta y por haber tenido una experiencia positiva. Por el contrario, y es un hecho que invita a la reflexión y al análisis, los dos CPR -centros con un solo docente- (1) son atendidos por maestros sin experiencia o con tan solo un curso escolar de experiencia, respectivamente; (2) los docentes asignados y directores aluden constantemente a la sensación de soledad, carga burocrática e incompetencia profesional para atender a alumnado con edades comprendidas entre los tres y los doce años, y (3) la trayectoria de estos centros se ha caracterizado por cambiar de maestro/a-director/a cada dos o tres cursos. 


\section{La complejidad de las lógicas en las aulas multinivel}

La diversidad es una característica inherente a cualquier práctica educativa, pero en las escuelas ubicadas en los entornos rurales cobra un especial sentido por las propias características contextuales -demográficas, físicas y naturales, económicas...- y educativas - dimensiones y capacidad del centro, características del alumnado y del profesorado, recursos...- inherentes a las aulas multigrado. En este sentido, una de las maestras comenta:

Trabajar con tan pocos alumnos te permite trabajar con una atención más individualizada [entrevista, C.G.R., directora y maestra de infantil].

De acuerdo con Boix (2011), el aula multigrado se caracteriza porque en ella se percibe "la diversidad dentro de la diversidad", pero hemos de tener en cuenta que "no se habla de diversidad en términos de discapacidad o dificultad de aprendizaje, sino de la heterogeneidad de las personas que conviven en una misma experiencia educativa" (García, Delgado y Pozuelos, 2017, p. 125), este hecho se constata en las palabras de una docente:

Puedes individualizar aspectos que le cuestan más trabajo a cada uno, lo puedes reforzar más, porque tienes más tiempo, el trabajo está más concentrado y puedes individualizar el aprendizaje [entrevista, M.E.E., maestra de educación infantil].

Como venimos señalando, entre las potencialidades de las aulas multigrados encontramos una baja ratio docente-discente, aspecto que favorece que el docente se adapte al nivel de competencia curricular de su alumnado, que se potencie una atención educativa personalizada (Boix, 2011), que se haga uso de estrategias de enseñanza y evaluación adaptadas a las características del alumnado (Abós y Boix, 2017) y que el docente disponga de mayor tiempo para atender al alumnado, favoreciendo la atención a la diversidad. En el aula multinivel la gestión de la heterogeneidad se vincula con la capacidad de los docentes de adaptar los procesos de enseñanza-aprendizaje a los cursos en los que su alumnado está adscrito (Boix y Bustos, 2014), por atender a alumnado con distintas edades, competencias y grados de conocimientos y por potenciar el rol del alumno/a tutor/a.

Las características organizativas de las aulas multigrado van a facilitar que emerja la figura del alumno/a-tutor/a (Boix, 2011) o alumno/a guía y orientador/a (Boix y Bustos, 2014), pues potencia el aprendizaje colaborativo entre iguales, como expresan algunos docentes:

Se ayudan mucho entre ellos, se conocen muy bien y yo como que los veo unos niños más felices [entrevista, M.A., maestra de francés y primaria].

Los alumnos avanzados ayudan a los niños más pequeños. Puedes trabajar mediante la figura del alumno-tutor [entrevista, F.A., maestro de educación física y primaria].

Este tipo de aprendizaje se constituye como una actividad informal, coyuntural, espontánea y no planificada en la cual "se aprovecha la capacidad y/o la competencia de mediador que puede tener un mismo alumnado para ayudar a sus compañeros en la 
adquisición de conocimiento/información” (Boix, 2011, p. 19), interactuando con compañeros de distinta edad (Ruiz y Ruiz, 2017), aspecto que favorece la relación entre iguales.

En este tipo de agrupamientos hay una unión muy especial entre ellos. Son unos niños que se cuidan mucho, pero no solo a nivel de aula, sino a nivel de centro, que los más mayores cuidan excesivamente de los más pequeños y se protegen mucho entre ellos [entrevista, C.G.R., directora y maestra de infantil].

Se enriquecen los pequeños de los grandes, ellos se enriquecen en todas las asignaturas, es muy bueno porque se ayudan unos a otros, además de forma espontánea, no necesitas decirle "iayúdalo!", el mayor le ayuda y le dice "mira esto es así, es así” [entrevista, B.L.P., directora maestra de inglés y primaria].

Estas acciones escolares y educativas se caracterizan porque (1) pueden ser ejercidas por cualquier alumno/a, (2) todos pueden aprender de todos (Boix y Bustos, 2014), (3) se producen de forma puntual (Bustos, 2013) y (4) van a desarrollar la autoestima, la responsabilidad y la consolidación de aprendizajes del alumnado-tutor (Boix, 2011), aspectos que van a potenciar el enriquecimiento académico del aula y el rendimiento escolar del alumnado.

Una de las particularidades del aula multigrado reside en que en ella se produce una convivencia inter-edades (Boix y Bustos, 2014), es decir, una situación educativa en la que los alumnos y alumnas aprenden unos de otros, como indican algunos docentes al respecto:

Los alumnos que son de un nivel menor captan y aprenden los contenidos del nivel superior [E.A., maestra de Educación Física y Primaria].

Al tener las unidades unidas por ciclos el alumnado del curso anterior aprenden ya cosas del curso siguiente [entrevista, L.P., directora y maestra de primaria].

En el aula se produce un aprendizaje por contagio debido a que el alumnado está en contacto con los contenidos curriculares de cursos superiores e inferiores de un modo continuo (Bustos, 2010), lo que les permite mayor amplitud de conocimientos en el aula (Boix y Bustos, 2014), contribuyendo a que la lógica de la fragmentación por edad se disipe.

En el caso de infantil al ser el aprendizaje globalizado, yo lo veo una ventaja porque al darlo todo globalizado cuando estás trabajando con los niños hay algunos que son capaces de coger contenidos que le estás enseñando a los niños de cinco años [entrevista, M.E.E., maestra de educación infantil].

Cuando das algún contenido todos los alumnos los aprenden a la misma vez. También hay que tener en cuenta que los alumnos de cursos superiores cuando terminan ayudan a los de cursos inferiores [grupo de discusión, R.R., maestro de primaria].

Bajo una óptica didáctica, las aulas multigrados se configuran como espacios compartidos en los que "circulan los saberes" (Santos, 2011) en una u otra dirección, 
aspecto que facilita que el alumnado mayor consolide conocimientos (Ruiz y Ruiz, 2017) y que el alumnado de menor edad esté expuesto a una serie de contenidos que no le corresponden por curso en el que está matriculado (Bustos, 2010).

\section{Conclusiones}

Como se desprende de los relatos docentes y en consonancia con otros trabajos (Abós, 2014; Boix, 2011; Bustos, 2006; Champollion, 2011; Feu, 2004; Juárez, Vargas y Vera, 2015; Juárez, 2017), las escuelas ubicadas en entornos rurales y, por lo tanto, las escuelas rurales multigrado adscritas a ellas, poseen una serie de potencialidades educativas -individualización del aprendizaje, mayor autonomía docente y capacidad de innovación, una ratio baja, aprendizaje colaborativo y por contagio, el apoyo de las familias...- que las dotan de una singularidad pedagógica y de ventajas educativas y sociales (Bustos, 2010) respecto a las escuelas urbanas. El aula multigrado se configura, además, como un espacio de aprendizaje cooperativo y reflexivo caracterizado por la heterogeneidad y por su capacidad de desarrollar estrategias de enseñanza-aprendizaje colaborativas, cooperativas e individualizadas (Bustos, 2010).

No obstante, y como venimos señalando, la docencia en el aula multigrado es una tarea compleja (Bustos, 2013) que requiere de una organización propia específica (Sepúlveda y Gallardo, 2011), de estrategias y metodologías educativas particulares (Quílez y Vázquez, 2012) y necesarias para el correcto desarrollo y atención educativa en un contexto educativo tan particular, y de una formación específica. Fruto de las evidencias aportadas en este trabajo se desprenden algunas de las debilidades del aula multigrado; a continuación, y junto a las aportaciones de otros trabajos, recogemos tres aspectos que a modo de propuestas de mejora creemos son fundamentales: la formación docente inicial y continua, la dotación de recursos materiales e infraestructuras y una mayor asignación de profesorado.

Uno de los aspectos más repetidos por los docentes se vincula con la falta de formación previa, en este sentido, la inexperiencia (Ruiz y Ruiz, 2017) y el desconocimiento de este tipo de centros y/o agrupamientos se posicionan como aspectos que provocan sensación de desánimo temporal e incertidumbre en los docentes durante los primeros meses, pues como señalan Hinojo, Raso e Hinojo (2010) de un modo general, los maestros y maestras no están formados de un modo específico para desarrollar su trabajo en escuelas rurales multigrado. En este sentido, son diversos los autores (Corchón, 2001, 2005; Corchón y Raso, 2004; Quílez y Vázquez, 2012) que señalan la importancia de una formación inicial y permanente específica relacionada con la escuela rural multigrado, pues como nos recuerda Boix (2007), los estudiantes de profesorado acaban su formación inicial desconociendo la escuela rural y formados para trabajar en una escuela completa y urbana. 
Para ello, y al igual que señalan los docentes que han participado en este trabajo, se demanda a las administraciones educativas, universidades y a los centros de profesorado -CEP- mayor implicación en la formación del profesorado -asignaturas, talleres, congresos, jornadas, cursos...- en relación con la atención educativa en aulas multigrado. Esta falta de formación se evidencia en un estudio realizado por Bustos (2014) en escuelas rurales y multigrado de Andalucía, Aragón y Cataluña en el que el $80.7 \%$ del profesorado afirma no haber participado en actividades de formación permanente relacionadas con el trabajo en grupos multigrados.

A esta situación, un tanto desoladora para los docentes sin experiencia en aulas rurales multigrado, se le une una demanda histórica relacionada con una mayor dotación de recursos materiales (Bustos, 2007) y humanos - responsabilidad de las administraciones educativas-, aspecto que se evidencia en otras investigaciones realizadas en países como México (Juárez, 2017), El Salvador (Juárez, 2017) o España (Feu, 2004) y que se posiciona como una de las grandes debilidades de las escuelas rurales multigrado. En este sentido, y mientras tanto el sistema de asignación de docentes como de recursos continúe bajo una lógica cuantitativa dotación económica/ asignación docente en función del número de alumnos y alumnas, estas necesidades y reivindicaciones persistirán.

Como venimos señalando, dimensiones como la falta de formación, la escasez de recursos humanos y materiales, la excesiva carga burocrática que soporta el profesorado de las escuelas rurales, entre otros aspectos, van a condicionar el tiempo de permanencia de los maestros y maestras en aulas rurales multigrados. Como se desprende de este trabajo, y a excepción de cuatro docentes, el tiempo de servicio prestado por el profesorado no excede de tres cursos académicos; esta información, que resulta significativa en cuanto a la permanencia del profesorado en escuelas rurales multigrado, coincide con el estudio de Bustos (2014) en escuelas rurales multigrado desarrollado en Andalucía, Aragón y Cataluña, en el que la media de años de servicio de profesorado en grupos rurales multigrado es de 5.6 y donde el $52.3 \%$ de los participantes acumulaban tres o menos de tres años de servicio en esta modalidad.

Es por todo lo señalado hasta el momento que entendemos que es vital hacer hincapié en el desarrollo de una epistemología de lo rural a la hora de concebir en cualquiera de las dimensiones constitutivas la escuela y la enseñanza rural. Entendemos, pues, que la invisibilidad de la escuela rural (Martínez y Bustos, 2011), ha de ser contrarrestada con más investigación (Bustos, 2011) y con trabajos y experiencias que destaquen sus fortalezas y debilidades (Abós, 2014), para, de este modo, contribuir a esa nueva construcción de conocimiento en y sobre una escuela rural que puede llegar a convertirse en un "laboratorio de renovación escolar" (Gallardo, 2011). 


\section{REFERENCIAS}

Abós, P. (2007). La escuela rural y sus condiciones: ¿tiene implicaciones en la formación del profesorado? Aula Abierta, 35(1), 83-90.

Abós, P. (2014). El modelo de escuela rural multigrado, ¿'es un modelo del que podamos aprender? ¿Es transferible a otro tipo de escuela? Innovación Educativa, 24, 99-118.

Abós, P., y Boix, R. (2017). Evaluación de los aprendizajes en escuelas rurales multigrado. Aula Abierta, 45(1), 41-48.

Boix, R. (coord.) (2004). La escuela rural: funcionamiento y necesidades. Barcelona: Praxis.

Boix, R. (2007). La escuela rural en Cataluña: problemáticas, propuestas y retos de futuro. Aula Abierta, 35(1), 77-82.

Boix, R. (2011). ¿Qué queda de la escuela rural? Algunas reflexiones sobre la realidad del aula multigrado. Profesorado. Revista de curriculum y formación del profesorado, 15(2), 13-23.

Boix, R., y Bustos, A. (2014). La enseñanza en las aulas multigrado: una aproximación a las actividades escolares y los recursos didácticos desde la perspectiva del profesorado. Revista Iberoamericana de Evaluación Educativa, 7(3), 29-43.

Bustos, A. (2007). Enseñar en la escuela rural aprendiendo a hacerlo. La evolución de la identidad profesional en las aulas multigrado. Profesorado. Revista de Currículum y Formación y del Profesorado, 11(3), 1-26.

Bustos, A. (2009). La escuela rural española ante un contexto en transformación. Revista de Educación, 350, 449-461.

Bustos, A. (2010). Aproximación a las aulas de escuela rural: heterogeneidad y aprendizaje en los grupos multigrado. Revista de Educación, 353, 353-378.

Bustos, A. (2011). Escuelas rurales y educación democrática. La oportunidad de la participación comunitaria. Revista Electrónica Interuniversitaria de Formación del Profesorado, 14(2), 105-114.

Bustos, A. (2013). El espacio y el tiempo en la escuela rural: algunas consideraciones sobre la didáctica multigrado. Investigación en la Escuela, 79, 31-41.

Bustos, A. (2014). La didáctica multigrado y las aulas rurales: perspectivas y datos para su análisis. Innovación educativa, (24), 119-131.
Cantón, I. (2004). Intervención educativa en la sociedad del conocimiento. Granada: Grupo Editorial Universitario.

Champollion, P. (2011). El impacto del territorio en la educación. El caso de la escuela rural en Francia. Profesorado. Revista de Currículum y Formación de Profesorado, 15(2), 53-69.

Corchón, E. (2000). Escuela rural: pasado, presente y perspectivas de futuro. Barcelona: Oikos Tau.

Corchón, E. (2001). La escuela rural andaluza. Granada: Consejo Escolar de Andalucía.

Corchón, E. (2005). La escuela en el medio rural: modelos organizativos. Barcelona: DaVinci Continental.

Corchón, E., y Raso, F. (2002). La escuela rural del siglo XXI: la educación del nuevo milenio. En M. L. Delgado et al. (eds.) (2002). Liderazgo educativo y escuela rural. Vol. II (pp. 1095-1100). Granada: Grupo Editorial Universitario.

Cornejo, M., Rojas, R. C., y Mendoza, F. (2008). La investigación con relatos de vida: pistas y opciones del diseño metodológico. Psykhe, 17, 29-39.

Cortés, P. C., González, B. A., y Sánchez, F. M. (2018). Agrupamientos escolares y retos para la educación inclusiva en infantil y primaria. Tendencias Pedagógicas, 32, 75-90.

Cortés, P., Simancas, E., y Mañas, M. (2018). Educación y comunicación. Una ecología del aprendizaje para la transformación de lo rural. En J. B. Martínez y E. Fernández, Ecologías del aprendizaje. Educación expandida en contextos múltiples (pp. 191-208). Madrid: Morata.

Departamento de Investigación y Documentación Parlamentaria (2015). Educación para poblaciones rurales en Latinoamérica: situación y marco normativo. Lima.

Feu, J. G. (2004). La escuela rural en España: apuntes sobre las potencialidades pedagógicas, relacionales y humanas de la misma. Revista Digital eRural, Educación, cultura y desarrollo rural, 2(3), 1-13.

Gallardo, M. (2011). La escuela de contexto rural. ¿De la diferencia a la desigualdad? Revista Iberoamericana de Educación, 55(5), 1-10.

García, F. J. P., Delgado, M. G., y Pozuelos, F. J. E. (2017). Atender a la diversidad en escuelas unitarias. Revista del Cisen Tramas/Maepova, 5(1), 123-139. 
González, B. A., Leite, A. E. M., y Rivas, J. I. R. (2018). La indagación educativa como herramienta de transformación social. En M. T. Castilla y V. Martín Solbes (coords.), Educación, derechos humanos y responsabilidad social (pp. 127-142). Barcelona: Octaedro.

Hinojo, F. J., Raso, F., e Hinojo, M. A. (2010). Análisis de la organización de la escuela rural en Andalucía: problemática y propuestas para un desarrollo de calidad. Revista Electrónica Iberoamericana de Calidad, Eficacia y Cambio en Educación, 8(1), 80-105.

Jiménez, J. (1983). La escuela unitaria. Barcelona: Laia.

Juárez, D. (2017). Percepciones de docentes rurales multigrado en México y El Salvador. Sinéctica, 49, 1-16.

Juárez, D., Vargas, P., y Vera, J. A. (2015). Condiciones de trabajo y prácticas didácticas de profesores que atienden escuelas primarias rurales en México. Revista Senderos Pedagógicos, 6, 15-27.

Junta de Andalucía (2003). Decreto 72/2003, de 18 de marzo, de Medidas de Impulso de la Sociedad del Conocimiento en Andalucía.

Leite, A. E. L. (2011). Historias de vida de maestros y maestras. La interminable construcción de las identidades: vida personal, trabajo y desarrollo profesional [Tesis doctoral]. Universidad de Málaga.

Martínez,J. B., y Bustos, A. (2011). Globalización, nuevas ruralidades y escuelas. Profesorado. Revista de Currículo y Formación del Profesorado, 15(2), 3-12.

Ministerio de Educación (2015). Temas de educación. Panorama de la Educación Rural en Argentina.

Ortega, M. A. (1995). La parienta pobre: significante y significados de la escuela rural. Madrid: Ministerio de Educación y Ciencia, CIDE.
Quílez, M., y Vázquez, R. M. (2012). Aulas multigrado o el mito de la mala calidad de enseñanza en la escuela rural. Revista Iberoamericana de Educación, 59(2), 1-12.

Rivas, J. I. F. (2014). Nuevas identidades en la formación del profesorado: la voz del alumnado. International Journal of Development and Educational Psychology, INFAD, 7(1), 487-494.

Rivas, J. I. F., Cortés, P. G., y Márquez, M. J. G. (2018). Experiencia y contexto: formar para transformar. En C. Monge y P. Gómez (eds.), Innovando la docencia desde la formación del profesorado (pp. 109-124). Madrid: Síntesis.

Ruiz, N. A., y Ruiz, J. R. G. (2017). Colegios rurales agrupados y formación universitaria. Profesorado, Revista de Currículum y Formación del Profesorado, 21(4), 205-230.

Santos, L. E. (2011). Aulas multigrado y circulación de los saberes: especificidades didácticas de la escuela rural. Profesorado. Revista de Currículum y Formación del Profesorado, 15(2), 71-91.

Sauras, P. (2000). Escuelas rurales. Revista de Educación, 322, 29-44.

Sepúlveda, M. P., y Gallardo, M. (2011). La escuela rural en una sociedad globalizada: nuevos caminos para una realidad silenciada. Profesorado. Revista de Currículum y Formación del Profesorado, 15(2), 142-153.

Showalter, D., Klein, R., Johnson, J., y Hartman, S. L. (2017). Why rural matters 2015-2016: Understanding the changing landscape. A Report of the Rural School and Community Trust. Rural School and Community Trust.

Stake, R. E. (1998). Investigación con estudio de casos. Madrid: Morata.

Veenman, S. (1984). Perceived problems of beginning teachers. Review of Educational Research, 54(2), 143-178.

Cómo citar este artículo:

González Alba, B., Cortés González, P., y Leite Méndez, A. (2020). Las aulas multigrado en el medio rural en Andalucía. Visiones docentes. IE Revista de Investigación Educativa de la REDIECH, 11, e-860. doi: 10.33010/ie_rie_rediech. v11i0.860. 